\title{
Nanomaterials design for engineering and biomedical applications
}

\author{
Vijaya K Rangari \\ Department of Materials Science and Engineering, Tuskegee University, Tuskegee-36088, Alabama, \\ USA. \\ Email: rangariv@mytu.tuskegee.edu
}

Nanoparticles are widely used because of their unique tunable properties and applications in automotive, electronic, textile, energy, aerospace and biomedical fields. The development and applications of nanoparticles derived from natural sources such as plant or animal are gaining more attention due to the high cost and environmental hazards of the petroleum and mineral derived products. This presentation covers the range of nanotechnology applications in polymer composite and biomedical fields.

Our recent work has been focused to design the nanoparticles using renewable waste resources such as waste eggshells to produce value added products like: a) Packaging, b) bone regeneration and c) drug delivery applications:

a). The extruded compostable (PBAT)/(PLA) blend films were reinforced with proteinaceous eggshell nanoparticles (PENP) ( 10 nm, Figure 1) engineered through mechanical attrition and ultrasound irradiation. The tensile analysis showed that the PENP led to enormous improvement in toughness of the ternary composites with 0.5 to $1.0 \%$ PENP, compared to the pristine blends systems.

b). The role of polymer-inorganic composite electrospun fibers as tissue engineering scaffolds is well recognized. We report here the fabrication of electrospun nanofibers from PLA with hydroxyapatite synthesized using eggshell. The ability of the composite electronspun nanofibers to support the attachment and growth of mammalian cells was examined by culturing the transformed ATCC CRL 11372 and SW480 cell line (ATCC CCL228 ) with the fibers in vitro. Cell attachment and growth were followed by light microscopy. The FE-SEM images revealed that the nanofibers were well-oriented and incorporated with the nanoparticles, which were distributed on the nanofibers. c). In this we studied these eggshell nanoparticles for Oral drug delivery systems which provide the most convenient, noninvasive, readily acceptable alternatives to parenteral systems. In this study, the eggshellderived calcium carbonate $\left(\mathrm{CaCO}_{3}\right)$ nanoparticles were used to develop enteric drug delivery system in the form of tablets. These nanoparticles were loaded with 5-fluorouracil as a model drug. Tablets with varying $\mathrm{CaCO}_{3}$ core and binder compositions were fabricated and coated with Eudragit S100 or Eudragit L100. Suitability for enteric delivery of the tablets was tested by oral administration to rabbits and radiography. Radiograph images showed that the tablet remained in the stomach of the rabbit for up to 3 hours. Further modifications of these biomaterial-derived nanoparticles and the coatings will enable manufacturing of stable formulations for slow or controlled release of pharmaceuticals for enteric delivery or tropical wound healing application.

Figure 1: Eggshell $\mathrm{CaCO}_{3}$ nanoparticles

\section{References}

1.Tarig A. Hassan, Vijay K. Rangari, Rohit K. Rana and Shaik Jeelani, Ultrasonics Sonochemistry, 20, 5, 1308-1315, 2013.

2.Tarig A. Hassan, Vijaya K. Rangari and Shaik Jeelani, ACS Sustainable Chemistry \& Engineering, 2014, 2 (4), 706-717. 